\title{
IOT -BASED APPROACH TO MONITOR PARKING SPACE IN Cities
}

\author{
Fatin Farhan Haque ${ }^{1}$, Weijia Zhou ${ }^{2}$, Jun-Shuo $\mathrm{Ng}^{3}$, Ahmed \\ Abdelgawad $^{4}$, Kumar Yelamarthi $^{5}$ and Frank Walsh ${ }^{6}$ \\ ${ }^{1,2,4,5}$ College of Science and Engineering, Central Michigan University, \\ Michigan, USA \\ ${ }^{3,6}$ Department of Computing and Mathematics, Waterford, Ireland
}

\begin{abstract}
Internet of Things is the next big thing, as almost everything developed now has an extensive use of data which is then used to get the daily statistics and usage of every individual. The work mainly consists of constructing a screen where the parking space will be shown, and a camera module will be set up, and PIR (Passive Infrared Sensor) will be at the entrance to detect the entrance of a car or any vehicle eligible to park at the lot. The vehicle will be scanned for its registration number in to provide a check whether the vehicle is registered to park or not. This also acts as the security of the parking lot. Moreover, a viable sensor will be placed at each parking slot through which the vacancy of each parking slot will be shown to determine the exact spot available to the user. In order to surpass the project completion, we will be using Raspberry Pi 3 with camera module mounted on it and by using Tensorflow, Node-Red we would be able to identify the car and the license number and also infrared sensor to detect the parking availability which would be displayed on the screen.
\end{abstract}

\section{KEYWORDS}

IoT, Node-Red, Tensor Flow, smart, parking

\section{INTRODUCTION}

The project proposal is initiated due to the current issues that are being faced by some regular people every day, i.e., to find a parking spot. The idea is mainly to create a device with a camera module mounted on it which will help to scan only the cars entering and exiting a parking lot and will display the appropriate space remaining in the lot. As it has been known that $20 \%$ of the drivers are getting irritated by driving the whole block to look for vacancy spot to park the car [12]. People are getting dependent upon the app which can help them get proper information about the parking lot occupancy, but inevitable due to the improper implication of GPS and other sensors [1]. The micro-controllers are used since they are battery-operated platform and costs very less with quite big life expectancy and also has numerous numbers of configuration options with a deployable architecture to run any application [2]. Another approach will show if the system is working according to the setup, if there are sufficient sensor network with whom the system is going to interact and process data that will help in showing the status i.e. occupied/vacancy of the parking lot [11]. If applied through IoT devices all the technological limitations can be portrayed as storage, processing and energy and the cloud will be able to deal with all the computation and real applications [7]. 
Some extra features have been added, and that is, the camera module will also scan the vehicle license number in order to determine whether the vehicle is registered for that space or not, this will help the authority to track down unregistered vehicle taking up space in the lot. Every system is not properly designed, as in terms of real-time detection some incorrect parking might lead to costly parking charges [3]. Through this system, time consumption will be minimized to find an available parking slot. The system can be applied in a different way, one is to create an algorithm and recognize the difference in walk-in state and drive state through which the state change will trigger the detection [4]. Another feature that can be added is with a viable sensor on each parking space, which will basically feed information to the cloud whether that space is empty in the lot or not and hence, a display of all the available space will be provided through which the vehicle entering can easily track down the space without wasting any time. As, it is known that the range of the sensor, for example, infrared lies from 20 to $4000 \mathrm{~mm}$ which will help us deduce the actual vacancy of the parking area [10].

Some previous work entitles as to use the visual sensor network in order to determine the parking space automatically and in order to do that encrypting of images or videos are being sent to the central controller which deals with all the decrypting and analyzing the contents that have been sent over. Implementing few techniques like detecting character recognition by OpenCV which stands for Optical Character Recognition and reading out the number plate number [8]. Using machine learning has been the next thing in today's world, which implies to unusual pattern in recognizing the parking spaces being allocated and dynamically checking out the vacancy in the parking lot area [9]. The sensors that can be used to achieve the task and get back with the information on the parking lot area are, ultrasonic, inductive loops, infrared sensors as the sensors tend to be very reliable but for huge parking lots the cost can be huge and hence using convolutional neural network can be an option to get the desired output [13]. The process and other related measures take enough power and capacity, but this can be resolute by connecting the network in the hub which in here acts in the cloud [14]. Another work relates to real-time detection of a vehicle entering and an automatic collection of parking fees which somehow gets declined if the real-time feature is not being focused on, as there have been already several literature reviews on the smart-parking system, hence other features are mostly being focused in.

\section{METHODOLOGY}

Two ways will be applied to make the work successful:

- Vehicle Recognition via Cameras: Multiple cameras will be installed in the parking lot to monitor all parking space and then accumulate their conditions.

- Viable Sensor: Each parking space is equipped with a viable sensor, which will basically feed information to the cloud whether space is empty or not.

- Provide any kind of warning or notification when any car is about to reserve the spot. Ultimately, a device with data receiver inside will be installed in the entrance to properly convert the information and present it to users in a straightforward way.

The challenges that can be faced while doing the project would be, mounting the cameras and getting proper feeds in the cloud and making a detection about vehicle's registration is also another challenge that needs to get overcome.

\section{RELATED WORKS}

The paper [5] illustrates the use of a smart parking facility with a few standard methods that are also IoT related and also for the ease of people in everyday life. Through an app, the registration 
and issuing of parking slip and vacancy are denoted and will be updated in the app through cloud mechanism. The sensors are used in an appropriate spot for the collection of data as per needed for the system to make necessary actions. It only deals with the space being empty and issuing the ticket as applicable.

The paper [6] discusses comparing two different paradigms: Compress-Then-Analyze (CTA) and Analyze-Then-Compress (ATC) when using a visual sensor system to analyze parking lot occupancy. CTA paradigm is to capture an image in real time and sending it to a processing unit to obtain information; ATC paradigm requires the visual content to be processed locally on the camera module itself before sending it off to be processed. The comparison is done to mainly compare energy consumption, bandwidth usage and, the accuracy of data of both paradigms. ATC method was much more effective on that when deploying in the real-world examples.

\section{IMPLEMENTATION}

A list of supplies that are needed for the smart parking lot are, Raspberry Pi 3 to convolute the results as per required, the Camera to scan the license plate number and recognizing the vehicle, OpenALPR to help in getting the correct the license plate number by using it's set of libraries, set of Wires for sensor connectivity, Toy Cars for the purpose of the real-time scenarios, Infrared Sensors for detecting the incoming vehicle approaching, LCD Screen for showing the exact spot to park the vehicle, PIR Sensor for detecting the motion of approaching car and working along the proposed way and lastly the LED to show that the oncoming vehicle is allowed to park in the available spot as it is registered in the system.

A Raspberry Pi 3 with camera module is mounted on the Pi (shown in Fig: 1) and connected with a display to helps us configure the related software and build-ups required in order to process the object identification with TensorFlow and the color of the object and also the model of the car will be identified. The Node-Red platform has been deployed which will focus on the checking of the license plate number and extracting the whole license number and then the number will be passed on to the cloud server which then will be used to check for the registration of the vehicle and through the check, if the license plate number matches up then the entrance gate will open up to let the vehicle in otherwise, it will stay close. In Fig 2, it has been shown how the Node-Red dashboard looks like after deployment. On another note, an offline process has also been applied which has been done by using the OpenALPR (open Automatic License Plate Recognition) which will in thus help to not rely on the internet as sometimes there might be delay or loss of connection. The code has been done on the Python platform and will help us determine the license plate number by using machine learning coding. The whole set up makes the work a pretty drastic since it takes a lot of time to configure the set of libraries and other related operations to make the Raspberry $\mathrm{Pi}$ suitable to detect the objects as per described in the tensorflow terminologies. There were few challenges that were faced while doing this, and it has been listed at a later stage in the paper.

The first step was to set up the tensorflow in the Pi and make it detect objects which later on we are going to focus on making it restricted to focus on only one object which is known as the single object detection. Fig 3 shows pictures of the data that we have collected, and which also shows a percentage of assumption of the object that the Pi was able to detect. In Fig 3, it can be seen that at the top-left corner of the detection frame, the accuracy of the object being detected and the object itself is illustrated. 


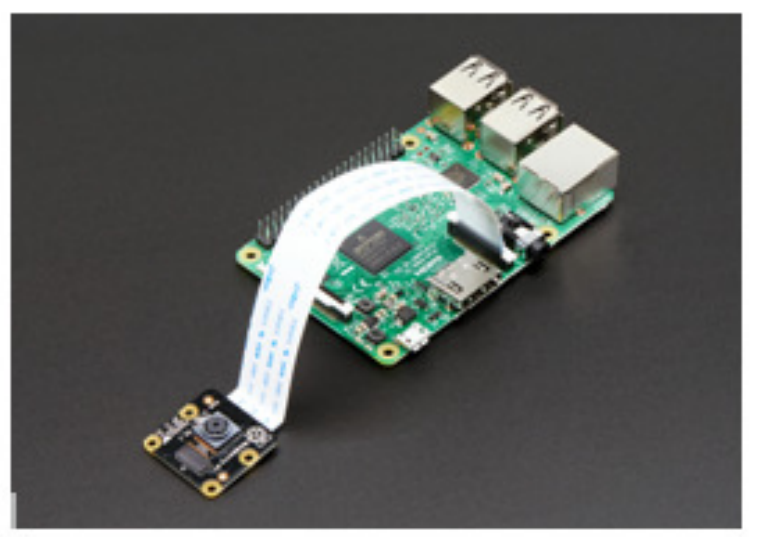

Fig 1: Raspberry Pi with the camera module

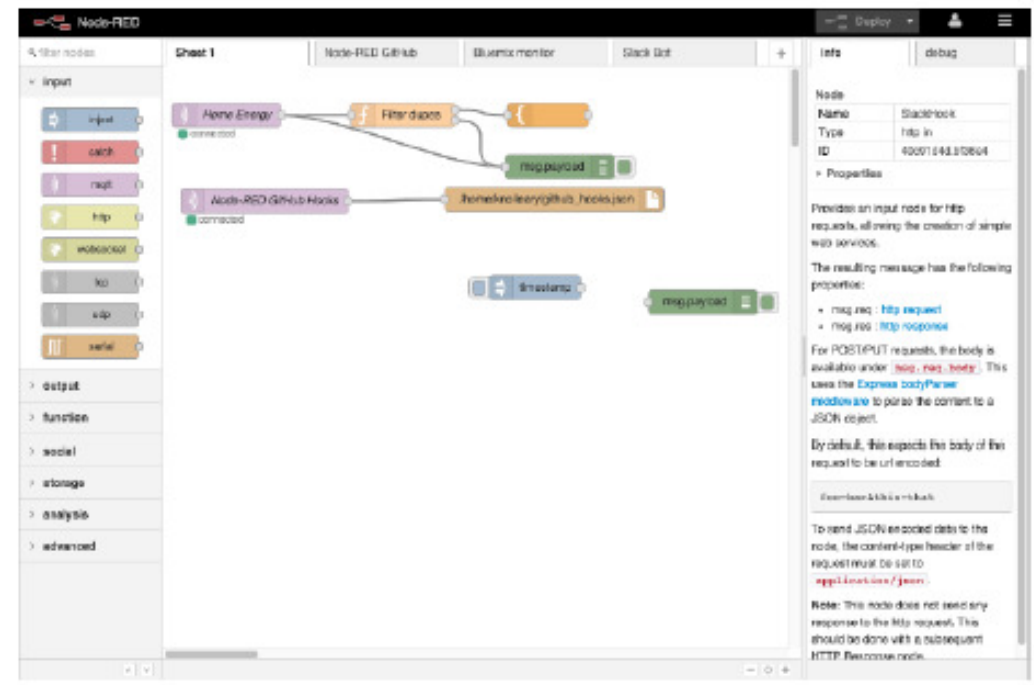

Fig 2: The Node-Red terminal where the action has been shown and the results received are displayed on the right-hand side.
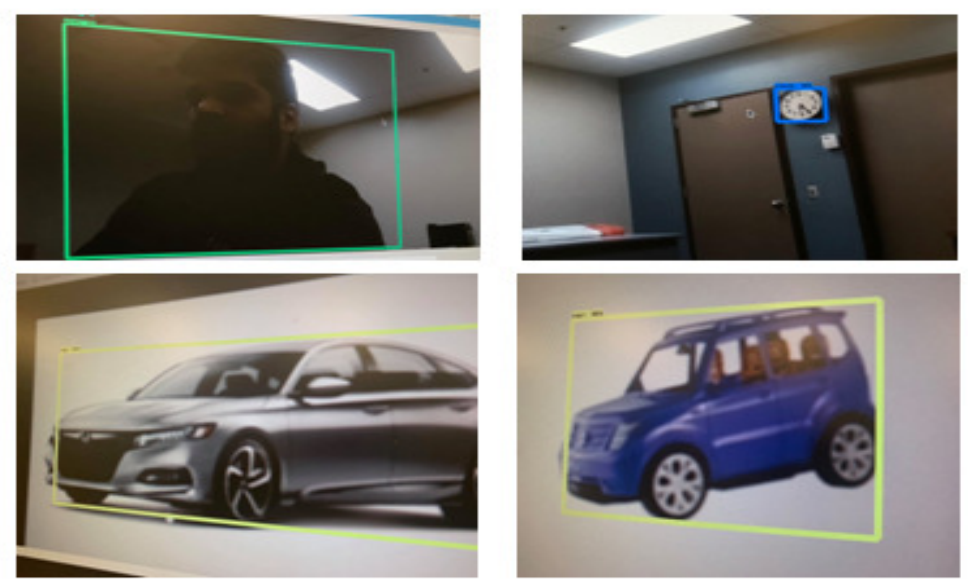

Fig 3: (a) a person with 95\% accuracy. (b) A clock with 95\% accuracy. (c) A car with 97\% accuracy. (d) A toy car with $98 \%$ accuracy. 
Fig 4 evaluates the flow of the process through multiple scanning and sensor reading and thus the results will be displayed on the screen as a vehicle approaches and try to get in the parking area.

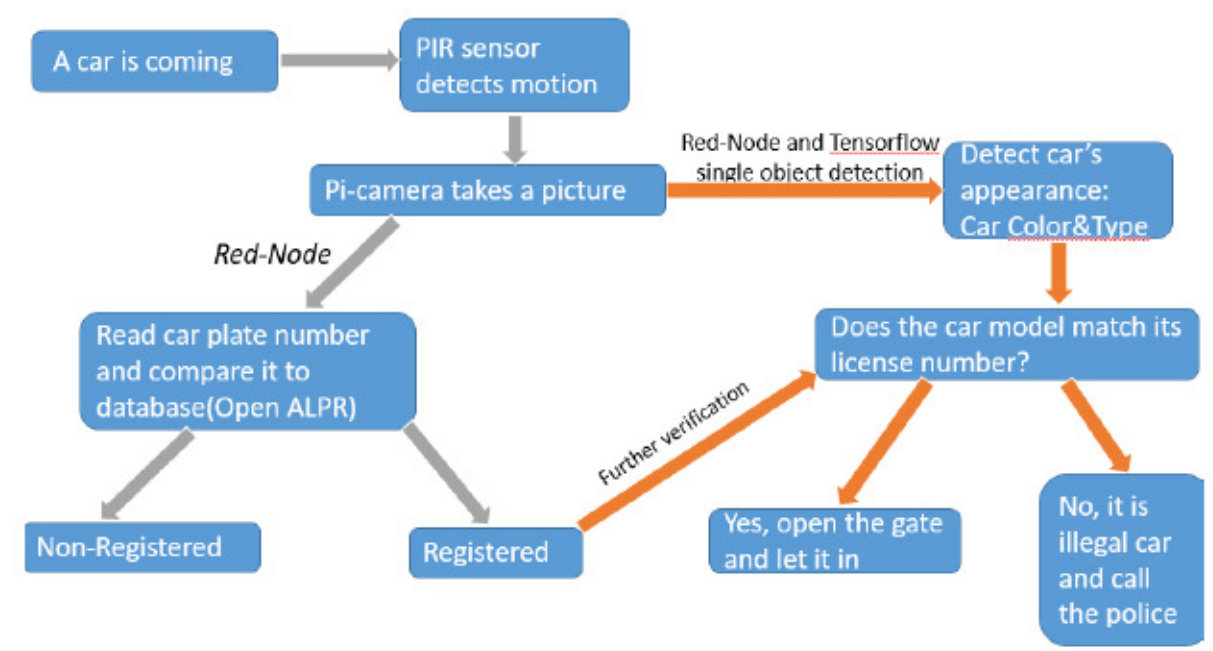

Fig 4: The flowchart describing te overall process.

Fig 5, shows the overall schematic diagram which had been hooked up on the breadboard using the sensor mentioned previously. This will be set up at the front gate in a box which in thus helps to resonate the program being deployed.

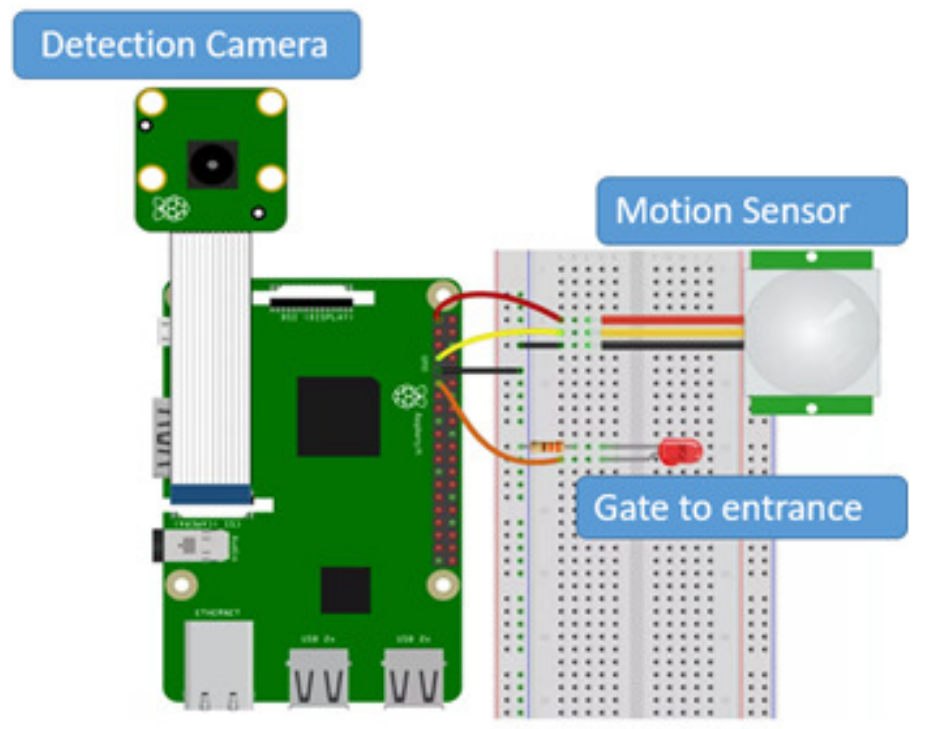

Fig 5: The schematic hook-up of the process.

In Fig 6, the flowchart depicts the use of the parking lot assignment and the screen demonstrates which parking area is vacant and where the vehicle can go and park, which is also in turns can be set as reserved for that vehicle. 


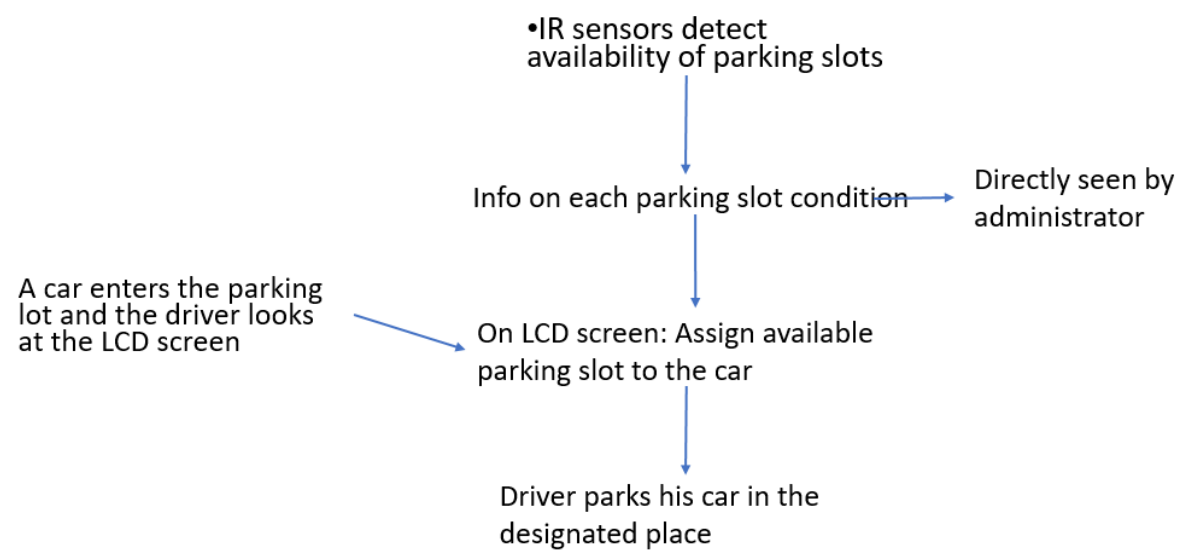

Fig 6: The flowchart representing the parking lot assignment.

\section{Results}

As the process has been deployed, we started to get data from the system. At first, we tried the license plate detection, and the results are shown in Fig 7, which portrays both the registered and unregistered car after reading the license plate number through the raspberry pi camera.

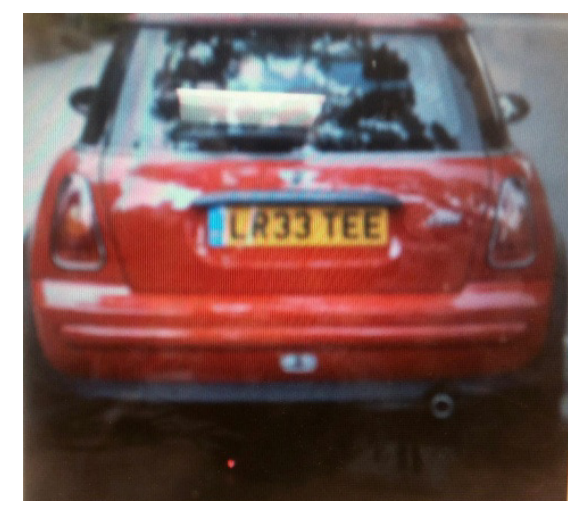

Fig 7: The picture illustrated the car license number detection and the license being recognized.
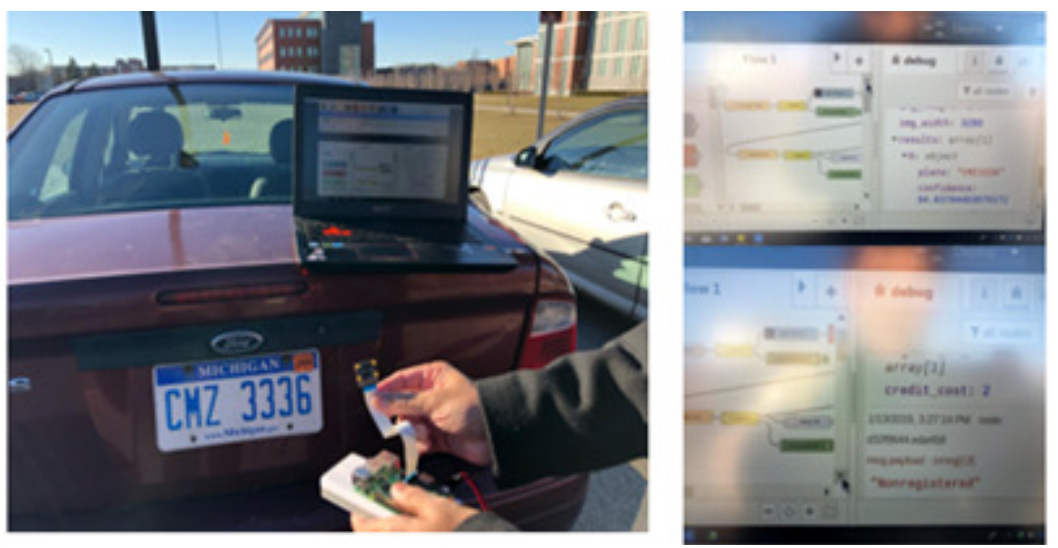

Fig 8: The picture shows the license of a different car and the license being recognized as unregistered. 
The hookup diagram and the LCD screen has been shown in Fig 8, which will save the user time to look for parking as it will automatically display which parking area to go in to since each parking slot is equipped with an infrared sensor and feeds information to the LCD.

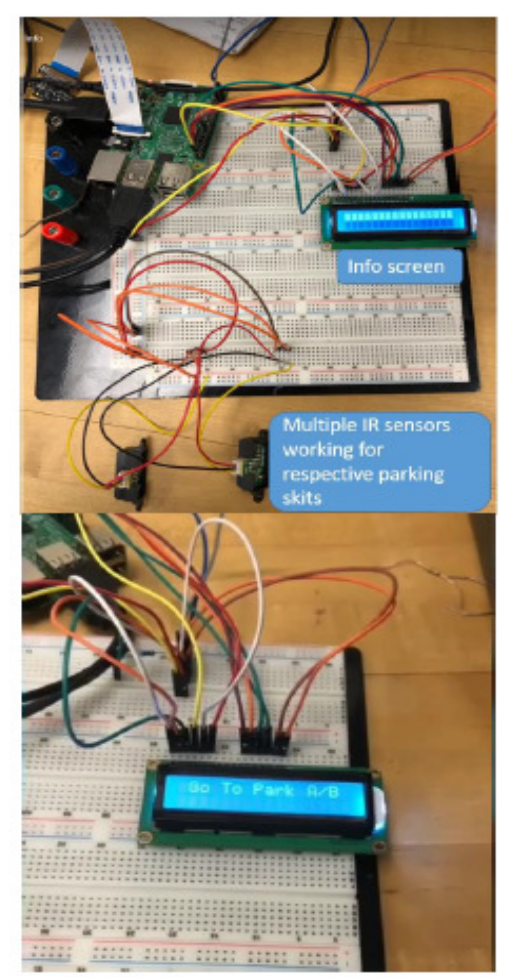

Fig 9: The hookup diagram and the anticipated output to be displayed.

\section{CONCLUSION AND FUTURE WORK}

The parking lot assignment has been the new thing which automatically tells users which parking slot to place their vehicle, as it has been one of the many concerns that everyone has nowadays, and everyone wants to park their vehicle more effectively and efficiently. The setup and everything else were done to ensure the ease of set up each parking lot equipment. The introduction of machine learning will help in determining the collection of vehicles coming in and will take less time in the future to detect the objects coming in.

Our future work lies in applying this to the real parking lot as this is just a prototype and there would be latency and power consumption, which we need to focus on making this embedded system further proceed.

\section{ACKNOWLEDGEMENTS}

The authors would like to thank K. Yelamarthi, F. Walsh and A. Abdelgawad for their constant feedback of the work and making it more keen and precise for the acquired data to make it more compatible in the realworld applications. 


\section{REFERENCES}

[1] A. Nandugudi, T. Ki, C. Nuessle \& G. Challen, 2014. PocketParker: pocketsourcing parking lot availability. In International Joint Conference on Pervasive and Ubiquitous Computing. Seattle, 2014, ACM.

[2] J. A. Propst, K. M. Poole \& J. O. Hallstrom, 2012. An embedded sensing approach to monitoring parking lot occupancy. In 50th Annual Southeast Regional Conference. Tuscaloosa, 2012. ACM.

[3] P. Sadhukhan, 2017. An IoT-based E-parking system for smart cities. In International Conference on Advances in Computing, Communications, and Informatics (ICACCI). Udupi, 2017. IEEE.

[4] S. Soubam, D. Banerjee, V. Naik, \& D. Chakraborty, 2016. BluePark: tracking parking and unparking events in indoor garanges. In International Conference on Distributed Computing and Networking. Singapore, 2016. ACM.

[5] V. Hans, P. S. Seithi, J. Kinra, 2015. An approach to IoT based car parking and reservation system on cloud. In International Conference on Green Computing and Internet of Things (ICGCIoT). IEEE.

[6] L. Baroffio, L. Bondi, M. Cesana, A. E. Redondi, M. Tagliasacchi, 2015, “A Visual Sensor Network for Parking Lot Occupancy Detection in Smart Cities". In IEEE 2nd World Forum on Internet of Things (WF-IoT). IEEE.

[7] A. Khanna, R. Anand, 2016.” IoT Based Smart Parking System”. In 2016 International Conference on Internet of Things and Applications (IOTA). IEEE.

[8] S. Rane, A. Dubey, T. Parida, 2017. "Design of IoT based intelligent parking system using image processing algorithms". In IEEE 2017 International Conference on Computing Methodologies and Communication (ICCMC).

[9] X. Ling, J. Sheng, O. Baiocchi, X. Liu, M. E. Tolentino, 2017. "Identifying Parking Spaces \& Detecting Occupancy Using Vision-based IoT Devices". In 2017 Global Internet of Things Summit (GIoTS).

[10] K. Laubhan, M. Trent, B. Root, A. Abdelgawad, K. Yelamarthi. “A Wearable Portable Electronic Travel Aid for Blind”. In 2016 International Conference on Electrical, Electronics, and Optimization Techniques (ICEEOT)

[11] S. Shinde, A. Patil, S. Chavan, S. Deshmukh, S. Ingleshwar. "IoT Based Parking System Using Google". In 2017 International Conference on I-SMAC (IoT in Socail, Mobile, Analytics and Cloud).

[12] S. Mendiratta, D. Debopam, D. R. Sona. “Automatic Car Parking System with Visual Indicator along with IoT".In 2017 International Conference on Microelectronic Devices, Circuits and Systems (ICMDCS).

[13] S. Valipour, M. Siam, E. Stroulia, M. Jagersand. "Parking-Stall Vacancy Indicator System, Based on Deep Convolutional Neural Networks". In 2016 IEEE 3rd World Forum on Internet of Things (WFIoT).

[14] K. Laubbhan, K. Talaat, S. Riehl, M. S. Aman, A. Abdelgawad, K. Yelamarthi. "A Low-Power IoT Framework: From Sensors to the Cloud". In 2016 IEEE International Conference on Electro Information Technology (EIT). 